Review

\title{
Non-Invasive Brain Stimulation for Insomnia - A Review of Current Data and Future Implications
}

Thierry Provencher ${ }^{1,2}$, Jonathan Charest ${ }^{1}$, Célyne H. Bastien ${ }^{1,2,3, *}$

1. École de Psychologie, Université Laval, Québec, QC, Canada; E-Mails: Thierry.provencher.1@ula val.ca; Jonathan.charest.2@ulaval.ca; celyne.bastien@psy.ulaval.ca

2. Centre de recherche CERVO, Québec, QC, Canada

3. Laboratoire de Recherche en Neurophysiologie Humaine et Sommeil, Québec, QC, Canada

* Correspondence: Célyne H. Bastien; E-Mail: celyne.bastien@psy.ulaval.ca

Academic Editor: Gerhard Litscher

Special Issue: Insomnia: Disorders of Initiation and Maintenance of Sleep

OBM Integrative and Complementary Medicine

2020 , volume 5 , issue 1

doi:10.21926/obm.icm.2001001
Received: October 09, 2019

Accepted: December 25, 2019

Published: January 02, 2020

\begin{abstract}
Neuroimaging evidences point to the role of hyperarousal in the pathophysiology of insomnia. While actual treatments fail to directly target brain hyperarousal, emergent complementary therapies known as neuromodulation techniques aim to improve sleep in people with insomnia by targeting irregularities in their brain activity. In this paper, we narratively review the most relevant studies reporting the application of neuromodulation techniques to improve sleep in individuals with insomnia. Using a non-systematic approach, we retrieved relevant literature across health-related bibliographic databases. Studies were included if they specifically assessed the effects of a neuromodulation technique on sleep in a sample of patients with insomnia. Three studies on transcranial direct current stimulation (tDCS) and six studies on repetitive transcranial magnetic stimulation (rTMS) were retained. No study on transcranial alternating current stimulation (tACS) was found. Preliminary data on tDCS in a sample of individuals with insomnia shows that targeting frontal regions may have a positive impact on sleep. Findings of rTMS studies, especially $1-\mathrm{Hz}$ low-frequency stimulation, suggest that it improves objective and subjective sleep in individuals with
\end{abstract}

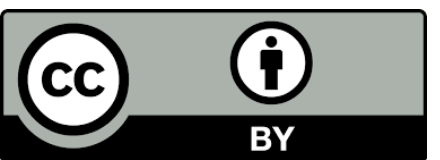

(C) 2020 by the author. This is an open access article distributed under the conditions of the Creative Commons by Attribution License, which permits unrestricted use, distribution, and reproduction in any medium or format, provided the original work is correctly cited. 
insomnia. Nonetheless, in both neuromodulation techniques, significant variability was found between stimulation parameters, study samples, and sleep outcomes. Although evidence on the impact of neuromodulation for insomnia remains scarce, recent data suggest it may have a sleep-deepening effect. Based on this review, and the limitations indicated by authors of included studies, we urge researchers to promote this field of research by testing different stimulation parameters, replicating already existing protocols, or adding standardized sleep-related outcomes.

\section{Keywords}

Repetitive transcranial magnetic stimulation; transcranial direct current stimulation; transcranial alternating current stimulation; insomnia; brain stimulation

\section{Introduction}

Insomnia is an important health issue [1], afflicting approximately $10 \%$ of the general population [2], and is associated with daily cognitive impairments (e.g., memory/attention deficits) [3] and higher rates of depression and anxiety [4]. The $5^{\text {th }}$ edition of the Diagnostic and Statistical Manual of Mental Disorders (DSM-5) [5] defines insomnia as a difficulty in initiating or maintaining sleep which is present at least three times a week in the past three months based on subjective reports [5]. It can present itself alone or in comorbidity with another health disorder (mental, physical, or sleep-related). Consensus is emerging in the scientific community that insomnia should only be diagnosed when an individual has adequate opportunity for sleep [6]. Conversely, sleep deprivation, defined as obtaining inadequate sleep to maintain adequate daytime alertness, is thought to have different causes and consequences $[7,8]$.

Many studies of insomnia disorder point to prevalent signs of hyperarousal [9-12]. However, inconsistencies can be found in neuroimaging findings in regard to this hyperarousal. For example, Nofzinger and colleagues [13] used $\left[{ }^{18} \mathrm{~F}\right.$ ] fluorodeoxyglucose (FDG) positron emission tomography (PET) to assess cerebral glucose metabolism in patients with insomnia and healthy subjects. Their results suggest a failure in the normal decline of glucose metabolism from wake to sleep state in individuals with insomnia. In other words, hyperarousal (i.e. increased glucose metabolism) in insomnia seems to remain present even during sleep. On the other hand, using a combination of polysomnography (PSG) and single-photon emission computerized tomography (SPECT) scan to assess and detect irregularities in cerebral blood flow, Smith and colleagues [14] have previously observed a state of hypoarousal during the first non-rapid eye movement (NREM) period across several brain regions in five patients with insomnia. However, as authors suggested, follow-up studies using various points across the NREM sleep cycle are still needed to evaluate how deactivation of structures possibly associated with slow wave sleep can be linked to hyperarousal. Recently, in light of the hyperarousal theory, researchers found that difficulty in falling asleep was associated with greater functional connectivity between primary sensory and supplementary motor regions as measured by resting-state functional magnetic resonance imaging (fMRI) [15]. Overall, although discrepancies have been found (e.g. increased glucose metabolism; NREM cerebral blood blow hypoarousal), most studies report an insomnia-specific state consistent with 
hyperarousal, usually associated to some extent with insomnia symptoms.

Individuals with insomnia also exhibit other neurobiochemical peculiarities as measured in neurophysiological and neurobiological studies. A structural neuroimaging synthesis by Riemann et al. [16] revealed a reduction in gray matter volume in several brain regions such as the medial frontal lobes, the dorsolateral prefrontal cortex, the parietal cortex and the anterior cingulate cortex. Research also shows that the decrease in bilateral hippocampi grey matter volume seems to be predominant in insomnia [17-19]. To this end, a study found that individuals with insomnia presented impaired memory and frontal lobe function, which eventually may be a contributing factor to the reduction in hippocampal volume [17]. Using fMRI, Santarnecchi et al. [20] demonstrated that poor sleep quality can induce a variety of changes in patients with sleep disorders [21-24], such as hypoactivation (i.e. decreased blow flow) in fronto-subcortical networks [25], associated with attention and memory impairments. An EEG study by Corsi-Cabrera et al. [26] also revealed that individuals with insomnia presented impaired frontal deactivation and disengagement of brain regions involved in executive control, attention, and self-awareness. This impairment in frontal control can be seen as a default in the 'top-down' (i.e. cortico-thalamocortical feedback loop) pathway of sleep-wake regulation. Using a transcranial magnetic stimulation (TMS) protocol, i.e. a technique used to assess brain physiology, van der Werf and colleagues [27] suggested that intracortical excitability might be a sustainable trait of individuals with insomnia. These authors delivered short-lived pulses of a strong magnetic field over the scalp of 16 individuals who were assigned either to Cognitive Behavioural Therapy for Insomnia (CBT-I) or a waitlist control group. Pre- and post-treatment results showed that even if subjective sleep onset latency (SOL) decreased and sleep efficiency (SE) increased, an increased in absolute excitability remained after treatment [27].

Thus, hyperarousal (and possibly increased excitability) remains a core component of insomnia and is thought to play a key role in its pathophysiology [28]. This can be observed clinically, in individuals with insomnia, as a cognitive focus on the insomnia and rumination about their sleep complaint [28]. Thus, clinical guidelines suggest that psychological treatment such as CBT-I, which aims at targeting dysfunctional cognitive beliefs and help individuals in enhancing their sleep hygiene while avoiding behaviors keeping them from sleeping well (e.g. drinking coffee before bedtime, etc.), should be used as first-line treatment whereas pharmacological treatments should be considered as second-line treatments [6]. Indeed, the American College of Physicians (ACP) actually recommends CBT-I as the initial treatment for chronic insomnia disorder [29]. Pharmacotherapy may be used on a short-term basis in shared decision making with the patient if the first-line treatment cannot be offered [6]. It should be considered only as an adjunct to CBT-I, and not the treatment of reference, for patients with insomnia disorder. To compensate the limitations of both pharmacological treatment (e.g. tolerance, adverse effects, and rebound insomnia after discontinuation) [30] and CBT-I (e.g. variability in sleep restriction therapy procedures) [31], complementary therapies have emerged in the aim of improving objective and subjective sleep quality and quantity in people with insomnia.

The term "non-invasive brain stimulation" (NIBS) has been coined to describe devices inducing electrical stimuli on the brain. Such methods are known for their role in modulating cortical excitability. Based on a NIBS umbrella model published by Liew and colleagues (2014) [32], there are two categories of NIBS: Transcranial Electric Stimulation (TES) and TMS. TMS techniques such as single-pulse or paired-pulse TMS can be used to assess brain physiology while other TMS 
techniques are rather known for their neuromodulation role: low or high-frequency repetitive TMS (rTMS), and patterned rTMS, also known as continuous or intermittent theta-burst stimulation (TBS).

On the other hand, TES techniques, used for their neuromodulation role, are subdivided into transcranial direct current stimulation (tDCS), transcranial alternating current stimulation (tACS) and transcranial random noise stimulation (tRNS). Although many techniques emerged in the last decades, tDCS, tACS, and rTMS received their share of empirical findings in sleep or sleep disorders.

The tDCS technique is a non-pharmacological treatment approach that offers the possibility of modulating cortical activity, which allows researchers and clinicians opportunities to target certain areas of the brain without undesirable effects [33, 34]. This technique is particularly interesting as it can lead to changes in brain activity in a non-invasive, safe and painless way [35]. Likewise, tACS aims at interfering with ongoing oscillations in the brain [36]. It is believed to impact neuronal membrane potentials by oscillatory electrical stimulation with specific or random frequencies and to interact with ongoing rhythmic cortical activities during sensory processing or cognitive processes [36, 37]. Lastly, rTMS is another method of stimulating the brain with little or no pain at the surface [38-40]. With rTMS, a magnetic field is generated with a magnetic coil placed above the scalp and the induced electrical stimulus activates neural elements in the cortex or subcortex white matter [40,41]. Ultimately, changes in excitability and activity in neurons and cortex have a central role in sleep [42]; tDCS, rTMS and tACS could be promising approaches for the treatment of various sleep disorders such as insomnia.

In our paper, we review the most relevant studies reporting the application of neuromodulation techniques (rTMS, tDCS, tACS) to improve sleep in individuals with insomnia. Although other TMS (e.g. TBS) and TES (e.g. tRNS) neuromodulation techniques may be relevant, these will not be the focus of this paper. Thus, we aim at providing a comprehensive perspective of the efficacy of neuromodulation and of parameters used in the past and still currently used.

\section{Materials and Methods}

This narrative review is not intended to be a systematic review. Therefore, we did not use a systematic approach. However, we retrieved relevant literature across the following electronic bibliographic databases: Cochrane Central Register of Controlled Trials (CENTRAL), PubMed, EMBASE, PsycINFO, and Web of Science (WoS). The terms "rTMS" (or: repetitive transcranial magnetic stimulation), "tES" (or: "transcranial electrical stimulation"), "tDCS" (or: "transcranial direct current stimulation") or "tACS" (or: "transcranial alternative current stimulation") were combined with "insomnia" using the "AND" operator. For each concept, we combined synonyms and Medical Subject Headings terms with the "OR" operator. Also, we performed a manual search of the reference lists of all relevant articles to identify potential studies for inclusion. Note that we limited our search to NIBS techniques categorised as "neuromodulation" techniques by Liew et al. [32]. Furthermore, a quick search using the terms "tRNS" (or: "transcranial random noise stimulation") and "TBS" (or: theta-burst stimulation), combined with "insomnia" using the "AND" operator, did not generate additional results.

We included studies assessing the effects of a neuromodulation technique (tDCS, tACS, rTMS) on the sleep of individuals suffering from insomnia. All sleep-related outcomes were included in 
our review, such as measures of objective (PSG) and subjective (sleep diaries, questionnaires) sleep architecture, as well as electroencephalography (EEG) and neuroimaging findings, when available. Because this field of research is not broad and we aimed to identify all relevant studies regardless of their publication type, we retained relevant conference and poster abstracts, even though they are usually excluded in reviews. Thus, we included three studies on tDCS ( 2 articles and 1 poster abstract), none on tACS, and six on rTMS ( 5 articles and 1 poster abstract).

\section{Results}

\subsection{Transcranial Direct Current Stimulation (tDCS) \& Insomnia}

tDCS is a non-invasive, painless, modality of brain stimulation, which modifies the "excitability" of cortical tissue following the application of weak direct current via electrodes [43]. tDCS uses a constant, low intensity current, delivered to a region or cerebral network of interest through two electrodes placed over the head [44]. Currently, researchers often report results of two types of stimulation with tDCS: "anodal" and "cathodal" stimulation. Anodal stimulation acts to excite neuronal activity while cathodal stimulation inhibits or reduces neuronal activity. The current flows from the anode to the cathode. While the cortical tissue underlying the anode (positive electrode) becomes depolarized and therefore more excitable; areas below the cathode (negative electrode) become hypopolarized and therefore less excitable. The magnitude of such membrane polarization changes is not sufficient in itself to directly trigger neurons [45]. Indeed, it can be used to manipulate the membrane potential of neurons and modulate spontaneous firing rates [44]. However, tDCS is insufficient on its own to discharge resting neurons or axons [46]. Still, its neuromodulating role may be of use to support the activation of the 'top-down' pathway of sleepwake regulation, known to be impaired in insomnia.

A sub-type of tDCS has been in emergence in the last decade: slow oscillatory-tDCS (so-tDCS). While it is essentially a tDCS protocol, others may argue that it falls in the range of tACS [47] (see Section 3.2 for more details on tACS). As indicated by Annarumma et al. (2018) [48], so-tDCS results in a polarized stimulation which benefits from both the effect of tDCS on cortical excitability and the synchronizing effect of tACS on the ongoing brain activity. This technique has been shown to modulate the EEG when it is applied simultaneously with a tACS-like sinusoidal waveform (0.75 $\mathrm{Hz}$ ) during NREM and rapid eye movement (REM) sleep [49, 50]. It was hypothesized that the application of so-tDCS would impact the spontaneous oscillatory activity of the stimulated cortical areas [51]. The rationale is that the generated sinusoidal waveform would be modulated by the external electric field, thus resulting in greater amplitude.

Results of three relevant studies of tDCS and insomnia are presented below. See Table 1 for study parameters (description of the sample, stimulation parameters, etc.), and Table 2 for the specific objective and subjective sleep outcomes of each study, and their principal findings. 
Table 1 Stimulation parameters in studies assessing the impact of tDCS on sleep in a sample of individuals with insomnia.

\begin{tabular}{|c|c|c|c|c|c|c|c|c|c|}
\hline $\begin{array}{l}\text { Author } \\
\text { (year) }\end{array}$ & Groups & $\begin{array}{l}\text { Age/sex } \\
\text { (insomnia) }\end{array}$ & Type of stimulator & Anode(s) & Cathode(s) & Stimulus intensity & Duration & Conditions & Protocol \\
\hline $\begin{array}{l}\text { Frase } \\
(2019) \\
{[42]}\end{array}$ & $\begin{array}{l}19 \text { patients } \\
\text { with } \\
\text { insomnia } \\
\text { (criteria: } \\
\text { DSM-5 \& } \\
\text { ICD-10) }\end{array}$ & $\begin{array}{l}43.8 \pm 15.1 \\
\text { years } \\
\text { (range 20- } \\
60 \text { years) } \\
13 \text { females } \\
\text { and } 6 \\
\text { males }\end{array}$ & $\begin{array}{l}\text { Battery-driven, } \\
\text { micro-processor- } \\
\text { controlled CE- } \\
\text { certified constant } \\
\text { current stimulator } \\
\text { (NeuroConn } \\
\text { GmbH; Illmenau, } \\
\text { Germany) }\end{array}$ & $\begin{array}{l}\mathrm{Fp} 1 / \mathrm{Fp} 2 \\
(5 \times 7 \mathrm{~cm})\end{array}$ & $\begin{array}{l}\mathrm{P} 3 / \mathrm{P} 4 \\
(10 \times 10 \\
\mathrm{cm})\end{array}$ & $1 \mathrm{~mA}$ & $\begin{array}{l}\text { Anodal }=2 \times 13 \\
\min (|S|: 20 \\
\min ) \\
\text { Cathodal }=2 \times \\
9 \text { min (ISI: } 20 \\
\min ) \\
\text { Sham }(2 \times 10 \\
\text { min) }\end{array}$ & $\begin{array}{l}\text { (1) Anodal; } \\
\text { (2) Cathodal; } \\
\text { (3) Sham }\end{array}$ & $\begin{array}{l}\text { Night } 1 \text { = Adaptation, } \\
\text { Night } 2 \text { = Baseline, } \\
\text { Night } 3 \text { = Sham, } \\
\text { Night } 4 \text { (1 week apart) } \\
\text { = anodal; } \\
\text { Night } 5 \text { (1 week } \\
\text { apart)= cathodal } \\
\text { (Nights 3, } 4 \text { and 5: } \\
\text { counterbalanced) }\end{array}$ \\
\hline $\begin{array}{l}\text { Saebipour } \\
(2015) \\
{[52]}\end{array}$ & $\begin{array}{l}6 \text { patients } \\
\text { with } \\
\text { insomnia } \\
\text { (criteria: } n / a \text { ) }\end{array}$ & $\begin{array}{l}34 \pm 7 \\
\text { years } \\
2 \text { females } \\
\text { and } 4 \\
\text { males }\end{array}$ & $\begin{array}{l}\text { Two-channel } \\
\text { battery-based } \\
\text { independent and } \\
\text { isolated } \\
\text { synchronized } \\
\text { stimulator } \\
\text { (Hidranco., } \\
\text { Tehran, Iran) }\end{array}$ & F3 \& F4 & Mastoids & $\begin{array}{l}\text { 1.33s/per cycle }(0.75 \mathrm{~Hz}) \\
\text { 1) } 0.00-0.33 \mathrm{~s}: 0 \text { to } 260 \mu \mathrm{A} \\
\text { 2) } 0.33-0.66 \mathrm{~s}: 260 \mu \mathrm{A} \\
\text { 3) } 0.66-0.99 \mathrm{~s}: 260 \text { to } 0 \mu \mathrm{A} \\
\text { 4) } 0.99-1.33 \mathrm{~s}: 0 \mu \mathrm{A}\end{array}$ & $\begin{array}{l}5 \times 5 \text { min } \\
\text { (after } \\
\text { establishment } \\
\text { of stage } 2 \text { or } \\
\text { deeper NREM } \\
\text { sleep) }\end{array}$ & $\begin{array}{l}\text { (1) so-tDCS; } \\
\text { (2) Sham }\end{array}$ & $\begin{array}{l}\text { Night } 1 \text { = Adaptation, } \\
\text { Night } \mathbf{2} \text { = Baseline, } \\
\text { Night } \mathbf{3} \text { = so-tDCS, } \\
\text { Night } 4 \text { (1 week apart) } \\
=\text { sham } \\
\text { (Nights } 3 \text { and 4: } \\
\text { counterbalanced) }\end{array}$ \\
\hline $\begin{array}{l}\text { Jung \& Jun } \\
\text { (2019) } \\
\text { - Poster } \\
{[53]}\end{array}$ & $\begin{array}{l}7 \text { patients } \\
\text { with } \\
\text { insomnia } \\
\text { (criteria: } n / a \text { ) }\end{array}$ & $\mathrm{n} / \mathrm{a}$ & $\mathrm{n} / \mathrm{a}$ & $\mathrm{n} / \mathrm{a}$ & $\mathrm{n} / \mathrm{a}$ & $\mathrm{n} / \mathrm{a}$ & $\mathrm{n} / \mathrm{a}$ & $\begin{array}{l}\text { (1) Anodal }(n=3) \text {; } \\
\text { (2) Cathodal } \\
(n=2) \text {; } \\
\text { (3) Sham }(n=2)\end{array}$ & $\mathrm{n} / \mathrm{a}$ \\
\hline
\end{tabular}

*DSM-5 = 5th edition of the Diagnostic and Statistical Manual of Mental Disorders; EEG = electroencephalography; ESS = Epworth sleepiness Scale; ICD-10 = 10th revision of the International Statistical Classification of Diseases and Related Health Problems; ISI = inter-stimulus interval; PSAS = Pre-Sleep Arousal Scale; PSG = polysomnography; PSQI = Pittsburgh Sleep Quality Index; tDCS = Transcranial direct current stimulation 
Table 2 Sleep outcomes and results from tDCS studies in a sample of individuals with insomnia.

\begin{tabular}{|c|c|c|}
\hline Author (year) & Sleep outcomes & Results \\
\hline Frase (2019) [42] & $\begin{array}{l}\text { Objective: PSG; EEG } \\
\text { Subjective: PSQI; ESS; PSAS; } \\
\text { Schlaffragebogen-A }\end{array}$ & $\begin{array}{l}\text { Non-significant tDCS effect: for all analyses, } p>0.1 \\
\text { - Sleep continuity (SOL, TST, SE, WASO, etc.) } \\
\text { - Sleep architecture (N2, N3, REM sleep) }\end{array}$ \\
\hline Saebipour (2015) [52] & $\begin{array}{l}\text { Objective: PSG } \\
\text { Subjective: Subjective rating of } \\
\text { restorative sleep (Likert scale) }\end{array}$ & $\begin{array}{l}\text { Non-significant tDCS effect (stimulation vs sham): } \\
\text { - Sleep-onset latency }(P=0.45, \mathrm{t} 5=0.818) \text {. } \\
\text { - Total sleep period }(\mathrm{P}=0.279, \mathrm{t} 5=1.213) \text {. } \\
\text { Significant tDCS effect (stimulation vs sham): } \\
\text { - Longer duration of } \mathrm{N} 3: 33 \pm 26 \mathrm{~min}(\mathrm{P}=0.026, \mathrm{t} 5=3.138) \\
\text { - Shorter duration of } \mathrm{N} 1: 22 \pm 17.7 \mathrm{~min}(\mathrm{P}=0.028, \mathrm{t} 5=-3.07) \\
\text { - Shorter duration of } \mathrm{N} 1 \text { and WASO (together): } 55.4 \pm 51 \mathrm{~min}(\mathrm{P}=0.045, \mathrm{t} 5 \\
=2.644) \\
\text { - Increased sleep efficiency (SE): } 9 \pm 7 \%(P=0.026, \mathrm{t} 5=3.13) \\
\text { - Increased average power at frequencies below } 1 \mathrm{~Hz} \text { after } 5 \text { min of } \\
\text { stimulation }(\mathrm{P} \leq 0.05 ; \text { Fig. } 5)\end{array}$ \\
\hline $\begin{array}{l}\text { Jung \& Jun (2019) } \\
\text { - Poster [53] }\end{array}$ & Objective or subjective: SE, TST, SOL & $\begin{array}{l}\text { Significant tDCS effect (1-month follow-up) } \\
\text { - Increased SE and TST in all patients (100\%) of the anodal condition, one } \\
\text { (50\%) of the cathodal group, and one (50\%) of the sham group } \\
\text { - Decreased SOL in two patients (67\%) of the anodal condition, one }(50 \%) \text { of } \\
\text { the cathodal group, and none }(0 \%) \text { of the sham group }\end{array}$ \\
\hline
\end{tabular}

${ }^{*}$ EEG = electroencephalography; ESS = Epworth Sleepiness Scale; PSAS = pre-sleep arousal scale; PSG = polysomnography; PSQI = Pittsburgh Sleep Quality Index; REM = rapid eye movement; SE = sleep efficiency; SOL = sleep onset latency; $\mathbf{t D C S}=$ transcranial direct current stimulation; $\mathbf{T S T}=$ total sleep time; WASO = wake after sleep onset 
Note that a group of researchers has published a review of tDCS in the context of sleep and insomnia [47], focusing on the potential use of tDCS in the treatment of insomnia. Furthermore, another group recently published a review providing a broad overview of studies in which brain stimulation has been applied to modulate sleepiness, sleep, and vigilance [48]. Of note, since tDCS current travels from the anode to the cathode, stimulation is always, technically, "anodal". Therefore, cathodal stimulation includes the same electrodes used in the anodal condition, although they are reversed. For instance, a F3 (anode)-P3 (cathode) anodal montage will become a P3 (anode)-F3 (cathode) cathodal montage. To avoid further confusion, we will report the original terms used by the authors in their respective studies.

\subsubsection{Frase et al. (2019) [42]}

In a within-subject repeated-measures sleep laboratory study, Frase et al. (2019) tested how bilateral frontal tDCS could influence total sleep time (TST) in 13 patients with insomnia, compared to sham stimulation (i.e. two blocks of 11 min tDCS with the stimulator fading in and out for 30s until it reached $1 \mathrm{~mA}$ at the beginning and end of each $11 \mathrm{~min}$ period with $20 \mathrm{~min}$ interval). They found that neither "anodal" (anodes = Fp1/Fp2 electrode sites in the 10-20 EEG system; cathodes = P3/P4 electrode sites in the 10-20 EEG system) nor "cathodal" (anodes =P3/P4; cathodes = Fp1/Fp2) stimulation modified TST. However, compared to cathodal and sham stimulation, they found that anodal tDCS led to a stronger decrease of TST in 19 healthy controls. Hence, authors concluded that the tDCS protocol did not modify sleep in patients with insomnia. Indeed, persistent hyperarousal in patients with insomnia might prevent sleep-promoting effects of bifrontal tDCS demonstrated in healthy controls [42].

\subsubsection{Saebipour et al. (2015) [52]}

In this paper, authors used a so-tDCS protocol. In a sham-controlled experiment (i.e. same procedure as the active condition) [52], so-tDCS (anodes = F3/F4 electrode sites in the 10-20 EEG system; cathodes = mastoids) at $0.75 \mathrm{~Hz}$ was applied after establishment of stage N2 (i.e. NREM2) or deeper NREM sleep in 6 patients with insomnia [52]. In terms of sleep architecture, although sleep onset latency (SOL) and TST did not differ between sham and stimulation, stage N1 (i.e. NREM1) duration was reduced ( $22 \pm 17.7 \mathrm{~min}$ ) and stage N3 (i.e. NREM3) duration ( $33 \pm 26 \mathrm{~min}$ ) was increased during so-tDCS. Also, sleep efficiency (SE) was reduced in so-tDCS $(9 \pm 7 \%)$. Regarding EEG power, 5 min of so-tDCS led to an increase in average power at frequencies below 1 $\mathrm{Hz}$, whereas sham did not. Thus, authors concluded that their pilot protocol had a sleep-deepening effect.

\subsubsection{Jung \& Jun (2019) [53]}

Published recently as a poster abstract, Jung \& Jun [53] reported the use of "anodal", "cathodal" and sham (i.e. no details) tDCS in a sample of 7 patients with insomnia. They found that TST and SE were improved with tDCS at 1-month follow-up in more than $50 \%$ of their sample in the "anodal" (2 patients out of 3 ) and "cathodal" (1 patient out of 2) groups, but not in the sham group (0 patient out of 2 ). Their preliminary results suggest that tDCS may indeed be effective in a sample of individuals with insomnia. Note that the information contained in the abstract did not 
allow us to extract the following information for Table 1: Anode(s), Cathode(s), Stimulus intensity, Duration, and Protocol. Furthermore, there was no mention whether sleep outcomes (i.e. TST, SE, SOL) were objective or subjective.

Possibly because frontal cortex impairments are seen in insomnia [17-19], tDCS protocols in the aforementioned studies mostly targeted frontal regions. However, findings regarding the efficacy of frontal tDCS are currently inconsistent. While fronto-parietal anodal and cathodal tDCS montages did not improve sleep in individuals with insomnia [42], a fronto-mastoids so-tDCS montage had a sleep-deepening effect [52]. These preliminary results suggest that when tDCS is applied in a sample of individuals with insomnia, sleep parameters are either increased or status quo. Indeed, no study has shown a deterioration of sleep after the intervention. Thus, until additional findings on tDCS applied to insomnia are published it may be considered to be somewhat beneficial at alleviating insomnia symptoms.

The equivocal efficacy of tDCS in insomnia may be the result of largely underpowered effects due to small sample sizes. Furthermore, one of the main factors that may be contributing to this reduction in statistical power may lie in the large between-group variation as reported on multiple occasions throughout the years in the tDCS domain [54-57]. For instance, using a similar stimulation protocol (motor-cortical protocol), differences up to $110 \%$ [57] and 184\% [58] were reported on the amplitude of motor evoked potentials (MEP). In this case, Nitsche et al. fixed an electrode over the representational field of the right abductor digiti minimi muscle (ADM) on the motor cortex and the other electrode contralaterally above the right orbit, using the device at 1 $\mathrm{mA}$ for the anodal (11 $\mathrm{min}$ ) and cathodal $(9 \mathrm{~min})$ conditions. In another study using anodal and cathodal stimulation during 20 minutes over the left primary motor cortex (M1), Tremblay and colleagues [59] reported one participant with an increased MEP amplitude of $251 \%$ (i.e. increased cortical excitability) and a second subject reporting a 41\% MEP amplitude decrease (i.e. decreased cortical excitability), thus giving grounds to the idea that high variability, in MEP amplitude or any other related outcome, can be found between groups, and especially within the same individual. Beyond small sample sizes and inter-subject variability also lies the intra-subject reliability. One major factor that is overseen in tDCS protocol is hair thickness. In other word, hair is the complete opposite of a conductive material, it is an insulator. It is suggested to keep the hair dry for better conductivity [60]. However, the high resistance of conductivity poses a problem in research settings. To overcome this issue, practitioners often use a large amount of saline water. Unfortunately, the saline water spread beyond the targeted point of measurement, thus leading towards unpredictable and undesirable direction [61]. Practitioners, or researchers, should be aware of these possible confounding factors in light of their significant effect on tDCS results.

Although results regarding the efficacy of tDCS in insomnia are unclear, they remain encouraging. Still, the exact mechanisms underlying its efficacy for insomnia are still poorly understood. Several hypotheses in regards to the enhancement of low frequency EEG activity have been suggested. For instance, targeting the frontal cortex with slow oscillatory stimulation may enhance the probability of action potentials occurring in prefrontal regions [62]. In other words, slow wave activity (SWA) may be increased. Low-frequency components in the EEG during NREM sleep (i.e. SWA) have been hypothesized to play a critical role in the reversion of cortical synaptic potentiation occurring during wakefulness [63], which is thought as a process through which sleep facilitates cognitive functions such as memory, learning, and sustained attention [64, 65]. Studies show that drugs (e.g. $G_{A B A}$ agonist) can induce SWA enhancement [66] and that this SWA 
increase may even be associated with an increased in sustained attention [67]. Thus, the first hypothesis as to the possible mechanism(s) of action of tDCS (and so-tDCS) is the idea that it induces the same SWA enhancement as sleep medication may do (without the undesirable chemical side effects). Notwithstanding, while the anode modulates the activity of the frontal cortex, the cathode may also induce an effect on the mastoid where it is located. In other words, in a fronto-mastoid montage for instance, while frontal stimulation may impact sleep, mastoid stimulation is also thought to have an effect. As such, another interesting hypothesis as to the efficacy of tDCS on sleep [68] lies in the possible effect of oscillatory stimulation applied to the mastoids [52]. Stimulation of the mastoid region (located near the ear) has been found to shorten sleep onset latency [69], leading to the idea that it may facilitate sleep. Additional findings indicate that stimulating the mastoids may also induce a galvanic response in the vestibular system. This tDCS-induced galvanic response is thought to mimic to some extent the vestibular impact of "rocking" on the EEG, hence leading to an increase in SWA [68]. In other words, SWA may be boosted by the stimulation of vestibular nerves (underneath the mastoid).

\subsection{Transcranial Alternating Current Stimulation (tACS) \& Insomnia}

While electric current in tDCS is considered direct, the generated current in tACS has an oscillation with a predetermined amplitude and frequency [36, 44]. In tACS protocols, an alternating current is applied (usually sinusoidal), inducing periodic shifts in the membrane potential, which leads to periodic alternating depolarizing and hyperpolarizing effects [48]. By a phenomenon called resonance, stimulation at a given frequency drives the cortical network to oscillate at the stimulation frequency with a greater amplitude [48].

In our literature search, no study met criteria for tACS besides the so-tDCS protocol of Saebipour and colleagues [52]. Indeed, their protocol may fit both tDCS and tACS protocols. Therefore, the very preliminary results suggest that so-tDCS induces a sleep-deepening effect [52]. That being said, we cannot know whether this effect is due to the cortical excitability modulation of tDCS, the synchronizing effect of tACS or a potentiation of both these effects.

Even though tACS in the context of insomnia has received limited focus, attempts were made to investigate how it can impact sleep. Although we cannot generalize findings from studies with good sleepers to a sample of individuals with insomnia, it may be relevant to present relevant studies in this field. Their results, exclusively based on samples of individuals who did not suffer from insomnia, are presented below.

Using, tACS, bilateral frontolateral slow waves $(0.75 \mathrm{~Hz})$ disrupted low frequency oscillation generation during an afternoon nap, which was correlated with declarative memory consolidation disruption [70]. Moreover, bilateral frontal slow wave $(0.5-1.2 \mathrm{~Hz})$ tACS led to an increase in slow wave and high-spindle power, as well as behavioral changes in long-term memory performance [71]. Lower gamma band $(40 \mathrm{~Hz})$ tACS during REM sleep applied on the frontal cortex influenced ongoing brain activity and induced self-reflective awareness in dreams [72]. In another experiment, using feedback-controlled $12 \mathrm{~Hz}$ tACS stimulation during sleep applied to the frontal and centro-parietal regions, no significant increase in declarative memory consolidation was found [73]. However, authors observed increased motor memory consolidation and sleep spindle activity [73]. Finally, in comparison with sham, bilateral central $14 \mathrm{~Hz}$ tACS led to an increased spindle density in central EEG site (Cz) [74]. However, as indicated by authors, it remains to be investigated 
how this enhancement in sleep spindle density is linked to a deeper sleep consolidation [74]. Overall, results suggest that tACS may have a sleep-deepening effect (i.e. increased sleep spindle density, increase in slow wave activity) and may play a role in memory performance and consolidation. Hence, preliminary data suggest that it is beneficial for sleep in healthy individuals.

In aforementioned studies, tACS protocols mostly targeted frontal [70-73] and central [73, 74] regions. Moreover, stimulation frequencies varied quite a lot. Researchers have targeted slow waves $[70,71]$, spindles $[73,74]$, or gamma activity [72]. The variability of these parameters renders a difficult interpretation of how tACS actually impacts sleep. However, a consistent finding is that the use of a specific oscillatory frequency (e.g. $1 \mathrm{~Hz}$ ) may indeed shape the underlying brain excitability so that it resonates at the same frequency (e.g. $1 \mathrm{~Hz}$ ). For instance, because lucid dreams are generally associated with high-frequency (gamma) activity in the frontal cortex [75], the induction of self-reflective awareness in dreams using a 40- $\mathrm{Hz}$-oscillation [72] represents a perfect example of how brain excitability can be trained. Although the results mentioned previously are interesting, we may not be able to generalize them to a sample of individuals with insomnia. Thus, knowing that tACS may have a positive impact on sleep (increase in SWA, sleep spindle density), future tACS studies should specifically target sleep in the context of sleep disturbances.

\subsection{Repetitive Transcranial Magnetic Stimulation (rTMS) \& Insomnia}

Repetitive Transcranial Magnetic Stimulation (rTMS) is a form of non-invasive brain stimulation that uses an electromagnetic coil placed on the scalp to create brief magnetic field pulses. Indeed, the coil generates a magnetic field inducing electrical currents in the underlying brain tissue beneath it as it passes through the skull $[40,76]$. While a single pulse $(<0.5 \mathrm{~ms})$ induces neuronal effects lasting only a fraction of a second, longer rTMS pulse sequences can cause prolonged neuroplastic changes that inhibit or excite the activity of the brain [40, 76]. High frequency rTMS (typically $\geq 10 \mathrm{~Hz}$ ) generally facilitates activation, whereas low frequency rTMS (usually $\leq 1 \mathrm{~Hz}$ ) is considered "inhibitory" [77].

Our literature review identified six relevant studies of rTMS and insomnia. These studies and their results are presented below. See Table 3 for study parameters (description of the sample, stimulation parameters, etc.), and Table 4 for the principal findings regarding the effects of rTMS on objective and subjective sleep outcomes, as well as cortical excitability. Note that a group of researchers has published a review of TMS and sleep disorders [78], focusing on the mechanisms underlying the generation of abnormal TMS measures. Although this fits outside the scope of this paper, we highlight its relevance to better understand the pathophysiology of sleep disorders, including chronic insomnia. Another group recently published a protocol for a systematic review on the effect of rTMS for insomnia [76]. Their results will be of high relevance to the field. 
Table 3 Stimulation parameters in studies assessing the impact of rTMS on sleep in a sample of individuals with insomnia.

\begin{tabular}{|c|c|c|c|c|c|c|c|c|c|}
\hline $\begin{array}{l}\text { Author } \\
\text { (year) }\end{array}$ & Groups & $\begin{array}{l}\text { Age/sex } \\
\text { (insomnia) }\end{array}$ & Type of stimulator & $\begin{array}{l}\text { Stimulation } \\
\text { sites }\end{array}$ & $\begin{array}{l}\text { Stimulation } \\
\text { frequency }\end{array}$ & Duration & $\begin{array}{l}\text { Stimulation } \\
\text { intensity }\end{array}$ & Conditions & Protocol \\
\hline $\begin{array}{l}\text { Feng } \\
(2019) \\
{[79]}\end{array}$ & $\begin{array}{l}32 \text { right-handed } \\
\text { patients with } \\
\text { insomnia } \\
\text { (criteria: ISCD-3) }\end{array}$ & $\begin{array}{l}44.8 \text { years } \\
(25-62) \\
20 \text { females } \\
\text { and } 12 \\
\text { males }\end{array}$ & $\begin{array}{l}\text { Commercially } \\
\text { available magnetic } \\
\text { stimulator (CCY-IV } \\
\text { model; YIRUIDE } \\
\text { Inc, Wuhan, Hubei } \\
\text { province, China) } \\
\text { with a 70-mm } \\
\text { figure-of-eight coil }\end{array}$ & $\begin{array}{l}\text { (1) Left } \\
\text { DLPFC } \\
\text { (2) Right } \\
\text { DLPFC }\end{array}$ & $\begin{array}{l}1 \mathrm{~Hz} \\
10 \mathrm{~s}(I S I=2 \mathrm{~s})\end{array}$ & $\begin{array}{l}30 \text { min } \\
(15 \text { min } \\
\text { over left } \\
\text { DLPFC, and } \\
15 \text { min } \\
\text { over right } \\
\text { DLPFC) }\end{array}$ & $\begin{array}{l}80 \% \text { of } \\
\text { rMT: } \\
\text { (resting } \\
\text { motor } \\
\text { threshold) }\end{array}$ & $\mathrm{n} / \mathrm{a}$ & $\begin{array}{l}1,500 \text { pulses. } \\
\text { The treatment was } \\
\text { applied once a day, } \\
5 \text { days per week } \\
\text { for } 2 \text {-week } \\
\text { treatment course } \\
\text { (with } 2 \text { days off } \\
\text { each weekend). }\end{array}$ \\
\hline $\begin{array}{l}\text { Jiang } \\
(2013) \\
{[80]}\end{array}$ & $\begin{array}{l}120 \text { right- } \\
\text { handed patients } \\
\text { with insomnia } \\
\text { (criteria: DSM- } \\
\text { IV) }\end{array}$ & $\begin{array}{l}\text { rTMS } \\
\text { group: } \\
48.31 \pm 8.45 \\
\text { years } \\
24 \text { females } \\
\text { and } 21 \\
\text { males }\end{array}$ & $\begin{array}{l}\text { Rapid } 2 \text { rTMS } \\
\text { stimulator } \\
\text { (Magstim, Wales, } \\
\text { UK). }\end{array}$ & Right DLPFC & $1 \mathrm{~Hz}$ & $30 \mathrm{~min}$ & $80 \%$ rMT & $\begin{array}{l}\text { (1) rTMS treatment } \\
\text { group ( } n=40 \text { ), } \\
\text { (2) medication } \\
\text { treatment group } \\
\text { ( } n=40): 2 \mathrm{mg} \\
\text { estazolam } \\
\text { (3) psychotherapy } \\
\text { group ( } n=40 \text { ): } 2 \text {-week } \\
\text { cognitive-behavioral } \\
\text { treatment }\end{array}$ & $\begin{array}{l}30 \text { pulses/string, } \\
\text { string interval } 2 \mathrm{~s} \text {, } \\
\text { total of } 60 \text { strings, } \\
\text { total stimulation } \\
\text { pulses } 1,800 \text {. } \\
\text { The treatment was } \\
\text { applied once a day } \\
\text { continuously for } 2 \\
\text { weeks. }\end{array}$ \\
\hline $\begin{array}{l}\text { Kunze } \\
\text { (2007) } \\
\text { - Poster } \\
{[81]}\end{array}$ & $\begin{array}{l}2 \text { patients with } \\
\text { insomnia } \\
\text { (criteria: } n / a \text { ) }\end{array}$ & $\mathrm{n} / \mathrm{a}$ & $\mathrm{n} / \mathrm{a}$ & $\begin{array}{l}\text { Patient 1: } \\
\text { left DLPFC } \\
\text { Patient 2: } \\
\text { right DLPFC }\end{array}$ & $\begin{array}{l}\text { Patient 1: } 10 \\
\mathrm{~Hz} \\
\text { Patient 2: } 1 \\
\mathrm{~Hz}\end{array}$ & $\mathrm{n} / \mathrm{a}$ & $\mathrm{n} / \mathrm{a}$ & $\mathrm{n} / \mathrm{a}$ & $\begin{array}{l}\text { Patient } 1 \text { : } \\
15 \text { sessions of } 10 \\
\text { Hz rTMS } \\
\text { Patient 2: } \\
15 \text { sessions of } 1 \mathrm{~Hz} \\
\text { rTMS. }\end{array}$ \\
\hline
\end{tabular}




\begin{tabular}{|c|c|c|c|c|c|c|c|c|c|}
\hline $\begin{array}{l}\text { Song } \\
(2019) \\
{[82]}\end{array}$ & $\begin{array}{l}20 \text { right-handed } \\
\text { individuals with } \\
\text { insomnia } \\
\text { (criteria: DSM-5) } \\
\text { and } 20 \text { healthy } \\
\text { controls }\end{array}$ & $\begin{array}{l}49.2 \pm 9.4 \\
\text { years } \\
8 \text { females } \\
\text { and } 12 \\
\text { males }\end{array}$ & $\begin{array}{l}\text { Rapid } 2 \text { rTMS } \\
\text { stimulator } \\
\text { (Magstim, Co. Ltd, } \\
\text { UK), with a 70-mm } \\
\text { figure-of-eight coil }\end{array}$ & $\begin{array}{l}\text { Right } \\
\text { posterior } \\
\text { parietal } \\
\text { cortex (P4) }\end{array}$ & $1 \mathrm{~Hz}$ & $34 \mathrm{~min}$ & $80 \%$ rMT & $n / a$ & $\begin{array}{l}3 \text { pulses/string; } \\
\text { string interval } 1 \mathrm{~s} ; \\
\text { total of } 500 \\
\text { strings; total } \\
\text { stimulation pulses } \\
1500 . \\
\text { The treatment was } \\
\text { applied once a day } \\
\text { continuously for } 2 \\
\text { weeks }\end{array}$ \\
\hline $\begin{array}{l}\text { Yu } \\
(2018) \\
{[83]}\end{array}$ & $\begin{array}{l}9 \text { right-handed } \\
\text { patients with } \\
\text { insomnia } \\
\text { (criteria: DSM- } \\
\text { IV) and } 9 \\
\text { healthy controls }\end{array}$ & $n / a$ & $\begin{array}{l}\text { Rapid } 2 \text { rTMS } \\
\text { system with figure- } \\
\text { of-eight coil } \\
\text { (Magstim } \\
\text { Company Ltd, } \\
\text { Whitland, U.K.) }\end{array}$ & $\begin{array}{l}\text { Neiguan } \\
\text { (PC6), } \\
\text { Shenmen } \\
\text { (HT7), and } \\
\text { Sanyinjiao } \\
\text { (SP6) } \\
\text { acupuncture } \\
\text { points } \\
\text { (acupoints) }\end{array}$ & $1 \mathrm{~Hz}$ & $6 \mathrm{~min}$ & $80 \%$ rMT & $n / a$ & $\begin{array}{l}\text { The treatment was } \\
\text { applied once a day } \\
\text { for } 3 \text { consecutive } \\
\text { days. }\end{array}$ \\
\hline $\begin{array}{l}\text { Zhang } \\
(2018) \\
{[84]}\end{array}$ & $\begin{array}{l}75 \text { patients with } \\
\text { insomnia } \\
\text { (criteria: DSM-5) }\end{array}$ & $\begin{array}{l}\text { Treatment } \\
\text { group: } \\
51.3 \text { years } \\
34 \text { females } \\
\text { and } 6 \text { males }\end{array}$ & $\begin{array}{l}\text { Magstim Rapid } 2 \\
\text { stimulator } \\
\text { (Magstim Co., UK), } \\
\text { with a figure-of- } \\
\text { eight-shaped coil }\end{array}$ & $\begin{array}{l}\text { Skull } \\
\text { overlying } \\
\text { the left } \\
\text { prefrontal } \\
\text { cortex }\end{array}$ & $1 \mathrm{~Hz}$ & $30 \mathrm{~min}$ & $100 \%$ rMT & $\begin{array}{l}\text { (1) Acupuncture } \\
\text { cooperated with } \\
\text { rTMS } \\
\text { (2) Acupuncture } \\
\text { cooperated with } \\
\text { sham rTMS }\end{array}$ & $\begin{array}{l}\text { Both groups were } \\
\text { given treatment } \\
\text { for } 4 \text { weeks with } 3 \\
\text { times a week. }\end{array}$ \\
\hline
\end{tabular}

${ }^{*}$ DLPFC $=$ Dorsolateral prefrontal cortex; DSM-5 = 5th edition of the Diagnostic and Statistical Manual of Mental Disorders; DSM-IV = 4th edition of the Diagnostic and Statistical Manual of Mental Disorders; ICSD-3 = 3rd revision of the International Classification of Sleep Disorders; ISI = inter-stimulus interval; rMT = Resting Motor Threshold; rTMS = Repetitive transcranial magnetic stimulation 
Table 4 Effects of rTMS on sleep outcomes and cortical excitability in studies with a sample of individuals with insomnia.

\begin{tabular}{|c|c|c|c|}
\hline \multirow{2}{*}{ Author (year) } & \multirow{2}{*}{ Outcomes } & \multicolumn{2}{|l|}{ Results } \\
\hline & & Sleep & Cortical excitability \\
\hline $\begin{array}{l}\text { Feng (2019) } \\
\text { [79] }\end{array}$ & $\begin{array}{l}\text { Objective sleep: none } \\
\text { Subjective sleep: PSQI } \\
\text { Cortical excitability: MEP }\end{array}$ & $\begin{array}{l}\text { Non-significant rTMS effect (post vs pre): } \\
\text { - No significant change in the ESS scale } \\
\text { Significant rTMS effect (post vs pre): } \\
\text { - Reduction of the PSQI score, } 10.94 \pm 2.58 \text { (post) vs } 15.84 \pm 3.72 \text { (pre) } \\
\text { after } 10 \text { days ( } p<0.0001 \text { ) }\end{array}$ & $\begin{array}{l}\text { Significant rTMS } \\
\text { effect (post vs pre): } \\
\text { - Reduction of the } \\
\text { left cortical } \\
\text { excitability (i.e. } \\
\text { lower MEP } \\
\text { amplitude), } 1.04 \pm \\
0.36 \mathrm{mV} \text { (post) vs. } \\
\text { 1.55. } \pm 0.42 \mathrm{mV} \\
\text { (pre), } \mathrm{p}=0.0008\end{array}$ \\
\hline $\begin{array}{l}\text { Jiang (2013) } \\
\text { [80] }\end{array}$ & $\begin{array}{l}\text { Objective sleep: PSG } \\
\text { Subjective sleep: PSQI } \\
\text { Cortical excitability: none }\end{array}$ & $\begin{array}{l}\text { Significant rTMS effect (after treatment vs before treatment): } \\
\text { - All studied parameters (SE, SOL, TST) significantly improved in all three } \\
\text { study groups }(p=0.05) \text { : Medication }>\text { rTMS }>\text { Psychotherapy }(p<0.05) \\
\text { - Increased Stage } 3 \text { and REM sleep: rTMS > Psychotherapy \& Medication } \\
\text { ( } p<0.05) \\
\text { - Decreased PSQI: rTMS > Psychotherapy \& Medication }(p<0.05)\end{array}$ & $\mathrm{n} / \mathrm{a}$ \\
\hline $\begin{array}{l}\text { Kunze (2007) } \\
\text { - Poster } \\
{[81]}\end{array}$ & $\begin{array}{l}\text { Objective sleep: One channel } \\
\text { electroencephalographic } \\
\text { sleep screening system } \\
\text { (QUISI). } \\
\text { Subjective sleep: Sleep } \\
\text { quality } \\
\text { Cortical excitability: none }\end{array}$ & $\begin{array}{l}\text { Significant rTMS effect (post vs pre): } \\
\text { - Reduced SOL } \\
\text { - Increased TST } \\
\text { - Increased REM latency }\end{array}$ & $\mathrm{n} / \mathrm{a}$ \\
\hline
\end{tabular}




$\begin{array}{ll}\text { Song (2019) } & \text { Objective sleep: EEG } \\ \text { Subjective sleep: PSQI; ISI; }\end{array}$

[82] ESS

Cortical excitability: none

Yu (2018)

[83]

\section{Objective sleep: EEG}

Subjective sleep: PSQI

Cortical excitability: none

\section{Significant rTMS effect:}

- Decline in PSQI, ESS and ISI scores after rTMS treatment and one

$\mathrm{n} / \mathrm{a}$ month after treatment

Significant rTMS effect (after stimulation vs before stimulation):

- Improvement in PSQI: 8.78 (before treatment) to 6.11 (after

$\mathrm{n} / \mathrm{a}$ treatment)

Non-significant rTMS effect (after treatment vs before treatment):

- No significant between-group difference in sleep diary derived SOL,

TST, WASO, and sleep quality, and daily dosage of hypnotics

- No significant between-group difference in actigraphy-derived SOL,

TST and WASO at 4th week post-treatment and 2nd week during follow-

up $(P>0.05)$

Objective sleep: Actigraphy

Zhang (2018) Subjective sleep: PSQI; ISI;

[84] Sleeping diary

Significant rTMS effect (after treatment vs before treatment):

- Reduction in ISI and PSQI scores at 4th week post-treatment and 2nd

Cortical excitability: none week during follow-up period, especially in patients in the treatment group $(\mathrm{P}<0.05)$

- Increase in SE at 4th week post-treatment and 2nd week during

follow-up period, especially in patients in the treatment group $(P<0.05)$

- Between-group difference in actigraphy-derived SE\% at 4th week post-

treatment and 2 nd week during follow-up $(P<0.05)$.

${ }^{*}$ EEG = electroencephalography; ESS = Epworth sleepiness scale; ISI = Insomnia Severity Index; MEP = motor evoked potential; PSG = polysomnography; PSQI = Pittsburgh Sleep Quality Index; REM = Rapid eye movement; $\mathbf{r T M S}=$ Repetitive transcranial magnetic stimulation; SE = Sleep efficiency; SOL = Sleep onset latency; TST = Total Sleep Time; $\mathbf{W A S O}=$ Wake After sleep Onset 


\subsubsection{Feng et al. (2019) [79]}

In a sample of thirty-two eligible right-handed patients with insomnia, low-frequency rTMS (1 $\mathrm{Hz}$ ) was applied over the left (15 min) and right (15 min) dorsolateral prefrontal cortex (DLPFC) for one session per day, 5 days per week during two weeks. No sham stimulation group was included in this study. The location of the left or right DLPFC was identified as being $5-\mathrm{cm}$ anterior to a primary motor cortex "hot spot" for producing the maximal motor response in the left or right abductor pollicis brevis (APB muscle). They found that the score on the Epworth Sleepiness Scale (ESS) remained stable before and after rTMS. On the other hand, Pittsburgh Sleep Quality Index (PSQI) scores were significantly reduced 10 days after the intervention. Furthermore, they observed a reduction of the MEP amplitude (i.e. reduced cortical excitability) after treatment, for the left DLPFC only. Thus, they concluded that sequential bilateral low-frequency rTMS over DLPFC improved subjective sleep quality in individuals with insomnia.

\subsubsection{Jiang et al. (2013) [80]}

In this study, 120 right-handed patients with an insomnia diagnosis according to the DSM-IV were allocated to one of three conditions: rTMS treatment group, medication treatment group, and psychotherapy group. Low-frequency rTMS $(1 \mathrm{~Hz})$ was applied over the right DLPFC for $30 \mathrm{~min}$, once a day, continuously for 2 weeks. No sham stimulation group was included in this study. The paper does not report how the location of the right DLPFC was identified. When comparing beforeafter effects in all three groups, they found that several PSG sleep parameters improved (SE, SOL, and TST) especially in the medication treatment group. Similar effects on sleep were observed in the rTMS treatment group. rTMS treatment was the most advantageous in improving N3 and REM sleep, as well as PSQI scores. Thus, rTMS provides an effective means of physical therapy for insomnia. As indicated by the authors, it is likely that rTMS will add to the treatment of chronic insomnia.

\subsubsection{Kunze et al. (2007) [81]}

Published as a poster abstract, two cases of individuals with insomnia were reported in this study: Patient 1 received 15 sessions of $10 \mathrm{~Hz}$ rTMS over the left DLPFC, Patient 2 received 15 sessions of $1 \mathrm{~Hz}$ rTMS over the right DLPFC. The abstract does not report how the location of the DLPFC was identified. Sleep improvements (reduced SOL, increased TST) were found in both patients. They also observed an increase in REM sleep latency. Their preliminary findings suggest that rTMS indeed improves sleep in individuals with insomnia.

\subsubsection{Song et al. (2019) [82]}

In this study, 20 right-handed individuals with insomnia as well as 20 healthy controls were recruited. Low-frequency rTMS $(1 \mathrm{~Hz})$ was applied over the right posterior parietal cortex (P4 electrode site in the 10-20 EEG system) for $34 \mathrm{~min}$, once a day, continuously for 2 weeks. No sham stimulation group was included in this study. They observed a significant decline in PSQI, ESS and ISI scores after rTMS treatment, as well as one month after treatment. In this study, authors evaluated the impact of rTMS on time-varying EEG network patterns. Before treatment, excessive information outflow in the left occipital, the frontal mid-line and right posterior temporal regions 
was found in individuals with insomnia compared with healthy controls. They found that rTMS targeting the patients' right parietal region increased information outflow in the left temporal region and decreased information outflow in the frontal mid-line region. Their study was the first to use low frequency rTMS to treat insomnia by stimulating the right posterior parietal region and was found to be effective to improve symptoms in a sample of individuals with insomnia.

\subsubsection{Yu et al. (2018) [83]}

Eighteen right-handed volunteers, 9 with insomnia and 9 healthy controls, received lowfrequency rTMS $(1 \mathrm{~Hz})$ for $6 \mathrm{~min}$, once a day, for three days, over the following acupuncture points, respectively located below the wrist on the inner forearm, on the wrist, and above the ankle: Neiguan (PC6), Shenmen (HT7), and Sanyinjiao (SP6). No sham stimulation group was included in this study. PSQI scores of individuals with insomnia decreased after rTMS. Specifically, improvements were seen in the bedtime, sleep efficiency and daytime functioning subcomponents of the PSQI. According to the authors, this is a direct evidence for the effect of magnetic stimulation.

\subsubsection{Zhang et al. (2018) [84]}

In this study, 75 subjects with DSM-5 insomnia diagnosis were allocated to one of two groups: acupuncture combined with rTMS (treatment group) or acupuncture combined with sham rTMS (control group; i.e. sham coil in the same area that was used for the motor cortex stimulation, and the stimulation parameters were set similarly). In the active group, participants received lowfrequency rTMS $(1 \mathrm{~Hz})$ over the left prefrontal cortex for $30 \mathrm{~min}, 3$ times a week, for 4 weeks. The paper does not report how the location of the left prefrontal cortex was identified. The authors examined the effect of acupuncture with/without rTMS on subjective (ISI, PSQI, sleep diaries) and objective (actigraphy) sleep parameters. They found that ISI and PSQI scores in both groups were significantly reduced after treatment, and compared to the control group, ISI scores had a significantly greater reduction in the treatment group, at least in the 4th week post-treatment and 2nd week during follow-up period. At those same time points, they also found that patients in the treatment group had significantly higher sleep efficiency (SE) (derived from the sleep diaries). Finally, no significant differences were found between groups in actigraphy-derived SOL, TST and wake after sleep onset (WASO), but SE was higher in the treatment group. Thus, when acupuncture conjoined with rTMS was used, it showed better improvements on subjective and objective measures of SE. Based on this study design, it is difficult to know which of acupuncture or rTMS potentiates the effect on sleep-related symptoms. Still, these findings suggest that active rTMS is better than sham rTMS at reducing insomnia symptoms.

Similarly to tDCS protocols, rTMS protocol mostly targeted frontal regions, especially the DLPFC. Specifically, studies assessed the impact of low-frequency rTMS over the right [79-81] or left DLPFC $[79,80]$, or high-frequency rTMS over the left DLPFC [81]. Other protocols have been used: lowfrequency rTMS over specific acupuncture points [83], or over the right posterior parietal cortex [82]. Although effects vary according to parameters in every protocol and every study has different outcomes, findings are somewhat consistent. rTMS was generally found to reduce PSQI, ESS and ISI scores, reduce SOL and increase TST, improve objective and subjective SE, and increase N3 and REM sleep. All those findings suggest that rTMS indeed improves sleep in individuals with 
insomnia. A question possibly worth asking is: why target the DLPFC? A shocking observation resulting from our review is that few authors actually explained the rationale behind their choice of stimulation target. Amongst those who did, the basic idea lies on the now-known fact that, from a neuroimaging perspective, individuals with insomnia may exhibit higher excitability in the DLPFC compared with healthy individuals [85]. Thus, in light with these findings, a significant therapeutic effect may specifically emerge from the inhibitory effect of low-frequency rTMS $(1 \mathrm{~Hz})$ on the DLPFC cortical excitability [79]. Although this result is encouraging, this study by Feng and colleagues [79] is the only rTMS study included in our paper that specifically assessed cortical excitability. Thus further research is still needed to adequately understand how this neuromodulation technique can actually regulate cortical excitability.

Years of experimental research with rTMS show that it may hold potential to improve sleep $[86,87]$. Hence, unsurprisingly, there has been an increasing interest in its application in the treatment of insomnia. The actual mechanisms of rTMS which could potentially lead to an improvement in sleep in insomnia individuals have not been completely identified. An interesting pathway though is that rTMS might be involved in the inhibition of the state of hyperarousal present within the cerebral cortex, therefore possibly modulating the underlying metabolic activity, including the synthesis and release of sleep-related hormones [28, 87]. It is believed that rTMS regulates brain plasticity by promoting the synthesis and release of brain-derived neurotrophic factor (BDNF) [88]. It has also been shown that rTMS promotes the release of gamma-aminobutyric acid (GABA) in neurons, a neurotransmitter most known for its inhibitory role $[89,90]$. Evidence shows that both BDNF and GABA are involved in sleep regulation [91], and that an increase in those endogenous chemicals may facilitate sleep onset or deeper sleep. Thus, the possible inhibition of the DLPFC by low-frequency rTMS (i.e. decreased cortical excitability) may result from changes in serum BDNF and GABA concentrations, which were also found to be associated with the improvement of insomnia symptoms [79].

\section{Discussion}

Based on the results of this review, we ascertain that the field of neuromodulation and insomnia is underdeveloped. Still, the majority of studies support the idea that neuromodulation (tDCS, rTMS, perhaps even tACS) can be effective in improving sleep or treating insomnia. First, aspects of sleep architecture may be improved with different protocols targeting frontal and parietal cortices: increased total sleep time, reduced sleep onset latency, etc. In addition, stimulation protocols improve brain activity by bringing it closer to what is considered normal, for example by increasing low frequency activity in the brain during sleep. That said, although sleeprelated improvements are often observed, few studies, perhaps none, assessed if participants perceived those changes as clinically significant.

Different mechanisms of action may explain the relative efficacy of tDCS and rTMS on sleep outcomes. This review revealed a possible efficacy of tDCS on sleep in individuals with insomnia, especially when using a so-tDCS protocol. However, only three studies were conducted, which greatly limits the interpretation of results. In the case of so-tDCS, a possible sleep-deepening effect has been observed, measured by an increase in average EEG power at frequencies below $1 \mathrm{~Hz}$. While its mechanisms of action have not been clearly identified, a dual-mechanism hypothesis has been formulated to explain how so-tDCS can possibly enhance slow wave activity: (1) stimulation 
of the prefrontal cortex leads to an increase in the probability of action potentials; (2) stimulation of the mastoids induces a galvanic vestibular response. Regarding rTMS (mostly low-frequency), its major role in the improvement of insomnia may lie in the inhibition of certain key structures known for their increased cortical excitability, such as the DLPFC. The magnetic pulse generated by the coil can potentially increase the synthesis and release of various sleep-promoting agents (BDNF; GABA). In sum, whether tDCS or rTMS is used, the rationale remains the same: the device must "inhibit" (or decrease the cortical excitability) brain regions exhibiting hyperarousal (i.e. increased cortical excitability).

This review illustrates a major finding in studies evaluating the effects of non-invasive brain stimulation on insomnia: the variability between protocols is unbounded. First of all, although most of the tDCS and rTMS protocols aimed at the frontal cortex, specifically the DLPFC, a few were interested in other areas: acupuncture points, parietal cortex etc. In addition, the duration of stimulation varied enormously, i.e. 3-15 treatment sessions (duration of 6-34 min) for rTMS, and 1 active session (duration of 18-26 $\mathrm{min}$ ) for tDCS. The sample sizes in tDCS studies were generally smaller than in rTMS, respectively 6-19 participants compared to 2-120 participants. Interestingly, tDCS studies ( 2 articles and 1 poster) did not include a control group, while the majority of rTMS studies involved either a control group or another treatment condition. Finally, neuromodulation studies present high variability on their sleep-related outcomes. Some studies have focused on how their stimulation protocol can alter the sleep architecture by using objective and subjective sleep measurements, which meets a certain clinical need. On the other hand, other studies have been interested in understanding how brain activity (e.g. EEG) is altered when using a technique known for its brain modulating effects (tDCS or rTMS).

A striking fact highlighted by this review is that it is difficult to put together the different results obtained by the aforementioned studies. What we notice, especially in Tables 1 and Table 3, is that the majority of research groups used different stimulation parameters: none of the presented protocol were identical. In other words, few (perhaps none) of the research groups attempted to replicate the protocol of another group. Thus, we are facing a paradox. Since the field of neuromodulation and insomnia has only begun to bloom, it is imperative to investigate the effects of various protocols on sleep. That said, it is also imperative to replicate certain protocols that have shown positive effects on sleep to study if those effects are still noticeable in a different sample of individuals who suffer from insomnia. This way, we can learn more about the real efficacy/effectiveness of tDCS, tACS and rTMS techniques to modulate the sleep of individuals suffering from insomnia. In addition, since all these protocols were not replicated, it is difficult to fully comprehend the inter-individual difference regarding effectiveness. Furthermore, placebo effect seems robust and significant, thus increasing the uncertainty of the optimal therapeutic regimen [87].

This review has limitations. First, we did not use a systematic approach during our study selection. Thus, we may have overlooked important results directly relevant to our research question. To limit this selection bias, we used a rigorous approach to retrieve relevant literature, using health-related bibliographic databases and performing a manual search of the reference lists of all relevant articles. In the end, we gathered essential papers on the effects of brain stimulation in insomnia. Second, we chose to limit this review to certain types of brain stimulation: tDCS, tACS, and rTMS. We selected those types because our initial literature review pointed to an increase in the publication of papers assessing the impact of these techniques on sleep. However, other types 
exist, and may be relevant for insomnia, such as transcranial random noise stimulation (tRNS) [92], or theta burst stimulation (TBS) [93]. Although those approaches in the field of neuromodulation are emerging, we invite other researchers to gather up evidence on their efficacy. Third, this review does not discuss the effects of stimulation on sleep in general but is rather specific to insomnia. We excluded papers that targeted sleep in non-insomnia samples, except for tACS protocols. We know, however, that some of the effects applied to sleep may or may not be generalized to the insomnia population (and not just good sleepers).

\section{Conclusions}

Based on our review, to promote the field of neuromodulation and insomnia, below are our recommendations for future purposes.

\subsection{Sample Size Calculation}

In the aforementioned studies, sample sizes vary between 1 and 120 individuals. Only a small portion of studies used a sample size calculation to predict how many participants should be included [84]. Since the main limitations in brain stimulation studies is the small sample size, we suggest that researchers in this field (and any other fields for that matter) should always include a sample size calculation. The number of participants should not be chosen at random but rather be based on quantitative criteria.

\subsection{Test Different Stimulation Parameters}

Frontal regions have been the main targets of brain stimulation for insomnia, both in tDCS and rTMS. Although this choice is often, if not always, based on empirical evidence, because of theoretical and clinical implication of the DLPFC and other related structures in insomnia [94, 95], testing different stimulation parameters would be indicated. As this field of research is still in its infancy, we have not yet explored all the potential targets of brain stimulation. It is worth further exploring whether other networks or brain structures could also benefit from stimulation. We hope that several studies exploiting different stimulation parameters (stimulus intensity, stimulation frequency, duration, stimulation sites, study conditions, etc.) will be published over the next few years, allowing the results to be synthesized in the form of a meta-analysis or other. The idea is that current studies do not show clearly how effective stimulation is and for whom, and in which context it should be used.

\subsection{Replicate Stimulation Protocols}

Although we recommend testing different parameters, it remains important to replicate protocols that have already been tested at least once, especially if they showed a positive impact on sleep parameters. As mentioned previously, replicating the same stimulation protocol in different samples will provide information about its true efficacy/effectiveness and will also allow us to get more data on inter-individual differences on efficiency. In other words, we may be more likely to answer the question: for whom the stimulation is more effective, and why? In doing so, we would obtain more clinical data, which is important, even necessary, for the choice of treatment. 


\subsection{Add Standardized Sleep-Related Outcomes}

Studies in the field of brain stimulation and insomnia have different outcomes, not always sleep-related. In order to gather up standardized evidence on the effects of different types of brain stimulation on insomnia symptoms, we recommend a minimal amount of sleep-related measures: Sleep architecture (duration of sleep stages, number of arousals, etc.), brain activity (EEG, MRI, etc.), symptoms of insomnia (PSQI, ESS, ISI), psychological functioning (Beck Anxiety Index or StateTrait Anxiety Index, Beck Depression Index, etc). In doing so, it will help the scientific community in understanding to extent to which the stimulation protocol actually impacts sleep.

\subsection{Report Safety Issues of the Stimulation Protocol}

Although stimulation has been declared safe when certain parameters are controlled [96-98], its safety should always be reported. The Food and Drug Administration (FDA) uses "safety" of a drug or other medical device as the first criterion before its approval, efficacy and effectiveness being irrelevant if the intervention is not considered safe for users [99]. Moreover, it is imperative to respect this process and therefore document the side and adverse effects of stimulation.

\subsection{Assessment of Individual Arousal in Participants}

There are discrepancies in the efficacy of different neuromodulation protocols, which may be explained by the level of arousal of an individual before the treatment session. We recommend that an arousal index be used prior to a tDCS (or rTMS) intervention to efficiently account for its impact on sleep. This could allow a categorization of participants that would be more sensitive to this kind of non-pharmacological approach. Moreover, differences in structure and electrical conductivity of the scalp, skull and neurons have been proposed to result in different susceptibility to applied currents, and variability of $\operatorname{tDCS}[46,100]$. To this end, computational modeling is increasingly being used to design electrode montages to calculate electric field strength and current flow direction in relation to neuronal orientation thus decreasing the variability in results $[100,101]$.

\section{Acknowledgments}

The authors would like to acknowledge the contribution of University Laval for providing the equipment used to conduct the bibliographic searches.

\section{Author Contributions}

Conceptualization and methodology: TP, JC, CHB;

Record screening/study selection: TP;

Data extraction: TP; JC

Writing - Original Draft: TP, JC, CHB;

Writing - Review and editing: TP, JC, CHB;

$\mathrm{TP}, \mathrm{JC}$, and $\mathrm{CHB}$ critically revised the article and approved the final version for submission. 


\section{Competing Interests}

The authors have declared that no competing interests exist

\section{References}

1. Collins PY, Patel V, Joestl SS, March D, Insel TR, Daar AS, et al. Grand challenges in global mental health. Nature. 2011; 475: 27-30.

2. Morin CM, LeBlanc M, Daley M, Gregoire J, Merette C. Epidemiology of insomnia: Prevalence, self-help treatments, consultations, and determinants of help-seeking behaviors. Sleep Med. 2006; 7: 123-130.

3. Wickens CD, Hutchins SD, Laux L, Sebok A. The impact of sleep disruption on complex cognitive tasks: A meta-analysis. Hum Factors. 2015; 57: 930-946.

4. Alvaro PK, Roberts RM, Harris JK. A systematic review assessing bidirectionality between sleep disturbances, anxiety, and depression. Sleep. 2013; 36: 1059-1068.

5. Association AP. Diagnostic and statistical manual of mental disorders (DSM- $5^{\circledR}$ ). 5th ed. Arlington, VA, US: American Psychiatric Pub; 2013.

6. Buysse DJ, Rush AJ, Reynolds CF. Clinical management of insomnia disorder. JAMA. 2017; 318: 1973-1974.

7. Orzeł-Gryglewska J. Consequences of sleep deprivation. Int J Occup Med Environ Health. 2010; 23: 95-114.

8. Pilkington S. Causes and consequences of sleep deprivation in hospitalised patients. Nurs Stand. 2013; 27: 35-42.

9. Dodds KL, Miller CB, Kyle SD, Marshall NS, Gordon CJ. Heart rate variability in insomnia patients: A critical review of the literature. Sleep Med Rev. 2017; 33: 88-100.

10. Drake CL, Pillai V, Roth T. Stress and sleep reactivity: A prospective investigation of the stressdiathesis model of insomnia. Sleep. 2014; 37: 1295-1304.

11. Healey ES, Kales A, Monroe $\amalg$, Bixler EO, Chamberlin K, Soldatos CR. Onset of insomnia: Role of life-stress events. Psychosom Med. 1981; 43: 439-451.

12. Morin $\mathrm{CM}$, Rodrigue $\mathrm{S}$, Ivers $\mathrm{H}$. Role of stress, arousal, and coping skills in primary insomnia. Psychosom Med. 2003; 65: 259-267.

13. Nofzinger EA, Buysse DJ, Germain A, Price JC, Miewald JM, Kupfer DJ. Functional neuroimaging evidence for hyperarousal in insomnia. Am J Psychiatry. 2004; 161: 2126-2128.

14. Smith MT, Perlis ML, Chengazi VU, Pennington J, Soeffing J, Ryan JM, et al. Neuroimaging of NREM sleep in primary insomnia: A Tc-99-HMPAO single photon emission computed tomography study. Sleep. 2002; 25: 325-335.

15. Killgore WD, Schwab ZJ, Kipman M, DelDonno SR, Weber M. Insomnia-related complaints correlate with functional connectivity between sensory-motor regions. Neuroreport. 2013; 24: 233-240.

16. Riemann D, Nissen C, Palagini L, Otte A, Perlis ML, Spiegelhalder K. The neurobiology, investigation, and treatment of chronic insomnia. Lancet Neurol. 2015; 14: 547-558.

17. Noh HJ, Joo EY, Kim ST, Yoon SM, Koo DL, Kim D, et al. The relationship between hippocampal volume and cognition in patients with chronic primary insomnia. J Clin Neurol. 2012; 8: 130138. 
18. Riemann D, Voderholzer U, Spiegelhalder K, Hornyak M, Buysse DJ, Nissen C, et al. Chronic insomnia and MRI-measured hippocampal volumes: A pilot study. Sleep. 2007; 30: 955-958.

19. Joo EY, Kim H, Suh S, Hong SB. Hippocampal substructural vulnerability to sleep disturbance and cognitive impairment in patients with chronic primary insomnia: Magnetic resonance imaging morphometry. Sleep. 2014; 37: 1189-1198.

20. Santarnecchi E, Del Bianco C, Sicilia I, Momi D, Di Lorenzo G, Ferrone S, et al. Age of insomnia onset correlates with a reversal of default mode network and supplementary motor cortex connectivity. Neural Plast. 2018; 2018: 3678534.

21. Altena $E$, Vrenken $H$, Van Der Werf YD, van den Heuvel OA, Van Someren EJ. Reduced orbitofrontal and parietal gray matter in chronic insomnia: A voxel-based morphometric study. Biol Psychiatry. 2010; 67: 182-185.

22. Stoffers D, Altena E, van der Werf YD, Sanz-Arigita EJ, Voorn TA, Astill RG, et al. The caudate: A key node in the neuronal network imbalance of insomnia? Brain. 2013; 137: 610-620.

23. Drummond SP, Walker M, Almklov E, Campos M, Anderson DE, Straus LD. Neural correlates of working memory performance in primary insomnia. Sleep. 2013; 36: 1307-1316.

24. Santarnecchi E, Sicilia I, Richiardi J, Vatti G, Polizzotto NR, Marino D, et al. Altered cortical and subcortical local coherence in obstructive sleep apnea: A functional magnetic resonance imaging study. J Sleep Res. 2013; 22: 337-347.

25. Spiegelhalder K, Regen W, Prem M, Baglioni C, Nissen C, Feige B, et al. Reduced anterior internal capsule white matter integrity in primary insomnia. Hum Brain Mapp. 2014; 35: 3431-3438.

26. Corsi-Cabrera M, Figueredo-Rodríguez P, del Río-Portilla Y, Sánchez-Romero J, Galán L, BoschBayard J. Enhanced frontoparietal synchronized activation during the wake-sleep transition in patients with primary insomnia. Sleep. 2012; 35: 501-511.

27. van der Werf YD, Altena E, van Dijk KD, Strijers RL, De Rijke W, Stam CJ, et al. Is disturbed intracortical excitability a stable trait of chronic insomnia? A study using transcranial magnetic stimulation before and after multimodal sleep therapy. Biol Psychiatry. 2010; 68: 950-955.

28. Riemann D, Spiegelhalder K, Feige B, Voderholzer U, Berger $M$, Perlis $M$, et al. The hyperarousal model of insomnia: A review of the concept and its evidence. Sleep Med Rev. 2010; 14: 19-31.

29. Qaseem A, Kansagara D, Forciea MA, Cooke M, Denberg TD. Management of chronic insomnia disorder in adults: A clinical practice guideline from the American College of Physicians. Ann Intern Med. 2016; 165: 125-133.

30. Trauer JM, Qian MY, Doyle JS, Rajaratnam SM, Cunnington D. Cognitive behavioral therapy for chronic insomnia: A systematic review and meta-analysis. Ann Intern Med. 2015; 163: 191-204.

31. Kyle SD, Aquino MRJ, Miller CB, Henry AL, Crawford MR, Espie CA, et al. Towards standardisation and improved understanding of sleep restriction therapy for insomnia disorder: A systematic examination of CBT-I trial content. Sleep Med Rev. 2015; 23: 83-88.

32. Liew SL, Santarnecchi E, Buch ER, Cohen LG. Non-invasive brain stimulation in neurorehabilitation: Local and distant effects for motor recovery. Front Hum Neurosci. 2014; 8: 378. 
33. Nitsche MA, Seeber A, Frommann K, Klein CC, Rochford C, Nitsche MS, et al. Modulating parameters of excitability during and after transcranial direct current stimulation of the human motor cortex. J Physiol. 2005; 568: 291-303.

34. Nitsche MA, Liebetanz D, Antal A, Lang N, Tergau F, Paulus W. Modulation of cortical excitability by weak direct current stimulation-technical, safety and functional aspects. Suppl Clin Neurophysiol. 2003; 56: 255-276.

35. Fregni F, Freedman S, Pascual-Leone A. Recent advances in the treatment of chronic pain with non-invasive brain stimulation techniques. Lancet Neurol. 2007; 6: 188-191.

36. Antal A, Boros K, Poreisz C, Chaieb L, Terney D, Paulus W. Comparatively weak after-effects of transcranial alternating current stimulation (tACS) on cortical excitability in humans. Brain Stimul. 2008; 1: 97-105.

37. Terney D, Chaieb L, Moliadze V, Antal A, Paulus W. Increasing human brain excitability by transcranial high-frequency random noise stimulation. J Neurosci. 2008; 28: 14147-14155.

38. Barker AT, Jalinous R, Freeston IL. Non-invasive magnetic stimulation of human motor cortex. Lancet. 1985; 325: 1106-1107.

39. Hallett M. Transcranial magnetic stimulation and the human brain. Nature. 2000; 406: 147150.

40. Ridding $M C$, Rothwell JC. Is there a future for therapeutic use of transcranial magnetic stimulation? Nat Rev Neurosci. 2007; 8: 559-567.

41. Diana M, Raij T, Melis M, Nummenmaa A, Leggio L, Bonci A. Rehabilitating the addicted brain with transcranial magnetic stimulation. Nat Rev Neurosci. 2017; 18: 685-693.

42. Frase L, Selhausen $P$, Krone L, Tsodor S, Jahn F, Feige B, et al. Differential effects of bifrontal tDCS on arousal and sleep duration in insomnia patients and healthy controls. Brain Stimul. 2019; 12: 674-683.

43. Meron D, Hedger N, Garner M, Baldwin DS. Transcranial direct current stimulation (tDCS) in the treatment of depression: Systematic review and meta-analysis of efficacy and tolerability. Neurosci Biobehav Rev. 2015; 57: 46-62.

44. Paulus W. Transcranial electrical stimulation (tES-tDCS; tRNS, tACS) methods. Neurosci Biobehav Rev. 2011; 21: 602-617.

45. Ruohonen J, Karhu J. tDCS possibly stimulates glial cells. Clin Neurophysiol. 2012; 123: 20062009.

46. Nitsche MA, Cohen LG, Wassermann EM, Priori A, Lang N, Antal A, et al. Transcranial direct current stimulation: State of the art 2008. Brain Stimul. 2008; 1: 206-223.

47. Rivera-Urbina GN, Nitsche MA, Molero-Chamizo A. Transcranial direct current stimulation (tDCS) in the context of sleep and insomnia. J Sleep Med Disord. 2016; 3: 1060-1065.

48. Annarumma L, D'Atri A, Alfonsi V, De Gennaro L. The efficacy of transcranial current stimulation techniques to modulate resting-state EEG, to affect vigilance and to promote sleepiness. Brain Sci. 2018; 8: E137.

49. Marshall L, Helgadóttir H, Mölle M, Born J. Boosting slow oscillations during sleep potentiates memory. Nature. 2006; 444: 610-613.

50. Marshall L, Kirov R, Brade J, Mölle M, Born J. Transcranial electrical currents to probe EEG brain rhythms and memory consolidation during sleep in humans. PloS One. 2011; 6: e16905.

51. Fröhlich F, McCormick DA. Endogenous electric fields may guide neocortical network activity. Neuron. 2010; 67: 129-143. 
52. Saebipour MR, Joghataei MT, Yoonessi A, Sadeghniiat-Haghighi K, Khalighinejad N, Khademi S. Slow oscillating transcranial direct current stimulation during sleep has a sleep-stabilizing effect in chronic insomnia: A pilot study. J Sleep Res. 2015; 24: 518-525.

53. Jung $K$, Jun J. Efficacy of transcranial direct-current stimulation on chronic insomnia. Brain Stimul. 2019; 12: 557.

54. Fricke K, Seeber AA, Thirugnanasambandam N, Paulus W, Nitsche MA, Rothwell JC. Time course of the induction of homeostatic plasticity generated by repeated transcranial direct current stimulation of the human motor cortex. J Neurophysiol. 2010; 105: 1141-1149.

55. Nitsche M, Fricke K, Henschke U, Schlitterlau A, Liebetanz D, Lang N, et al. Pharmacological modulation of cortical excitability shifts induced by transcranial direct current stimulation in humans. J Physiol. 2003; 553: 293-301.

56. Nitsche MA, Grundey J, Liebetanz D, Lang N, Tergau F, Paulus W. Catecholaminergic consolidation of motor cortical neuroplasticity in humans. Cereb Cortex. 2004; 14: 1240-1245.

57. Nitsche MA, Liebetanz D, Schlitterlau A, Henschke U, Fricke K, Frommann K, et al. GABAergic modulation of DC stimulation-induced motor cortex excitability shifts in humans. Eur J Neurosci. 2004; 19: 2720-2726.

58. Nitsche MA, Kuo MF, Karrasch R, Wächter B, Liebetanz D, Paulus W. Serotonin affects transcranial direct current-induced neuroplasticity in humans. Biol Psychiatry. 2009; 66: 503508.

59. Tremblay $S$, Beaulé $V$, Lepage JF, Théoret $H$. Anodal transcranial direct current stimulation modulates GABAB-related intracortical inhibition in the $M 1$ of healthy individuals. Neuroreport. 2013; 24: 46-50.

60. Feughelman M. Mechanical properties and structure of alpha-keratin fibres: Wool, human hair and related fibres. UNSW press; 1997.

61. Horvath JC, Carter O, Forte JD. Transcranial direct current stimulation: Five important issues we aren't discussing (but probably should be). Front Syst Neurosci. 2014; 8: 2.

62. Murphy M, Riedner BA, Huber R, Massimini M, Ferrarelli F, Tononi G. Source modeling sleep slow waves. Proc Natl Acad Sci USA. 2009; 106: 1608-1613.

63. Tononi G, Cirelli C. Sleep and synaptic homeostasis: A hypothesis. Brain Res Bull. 2003; 62: 143-150.

64. Huber R, Ghilardi MF, Massimini M, Tononi G. Local sleep and learning. Nature. 2004; 430: 78-81.

65. Stickgold R, Hobson JA, Fosse R, Fosse M. Sleep, learning, and dreams: Off-line memory reprocessing. Science. 2001; 294: 1052-1057.

66. Walsh JK, Deacon S, Dijk D-J, Lundahl J. The selective extrasynaptic GABAA agonist, gaboxadol, improves traditional hypnotic efficacy measures and enhances slow wave activity in a model of transient insomnia. Sleep. 2007; 30: 593-602.

67. Walsh JK, Randazzo AC, Stone K, Eisenstein R, Feren SD, Kajy S, et al. Tiagabine is associated with sustained attention during sleep restriction: Evidence for the value of slow-wave sleep enhancement? Sleep. 2006; 29: 433-443.

68. Cellini N, Mednick SC. Stimulating the sleeping brain: Current approaches to modulating memory-related sleep physiology. J Neurosci Methods. 2019; 316: 125-136. 
69. Krystal AD, Zammit GK, Wyatt JK, Quan SF, Edinger JD, White DP, et al. The effect of vestibular stimulation in a four-hour sleep phase advance model of transient insomnia. J Clin Sleep Med. 2010; 6: 315-321.

70. Garside P, Arizpe J, Lau C-I, Goh C, Walsh V. Cross-hemispheric alternating current stimulation during a nap disrupts slow wave activity and associated memory consolidation. Brain Stimul. 2015; 8: 520-527.

71. Ketz N, Jones AP, Bryant NB, Clark VP, Pilly PK. Closed-loop slow-wave tACS improves sleepdependent long-term memory generalization by modulating endogenous oscillations. J Neurosci. 2018; 38: 7314-7326.

72. Voss U, Holzmann R, Hobson A, Paulus W, Koppehele-Gossel J, Klimke A, et al. Induction of self awareness in dreams through frontal low current stimulation of gamma activity. Nat Neurosci. 2014; 17: 810-812.

73. Lustenberger C, Boyle MR, Alagapan S, Mellin JM, Vaughn BV, Fröhlich F. Feedback-controlled transcranial alternating current stimulation reveals a functional role of sleep spindles in motor memory consolidation. Curr Biol. 2016; 26: 2127-2136.

74. Bouchard M, Fortin-Langelier E, Frenette S, Cyr G, Latreille C, Lina J, et al. Is transcranial alternating current stimulation (tACS) an effective tool to entrain spindles during sleep in older individuals? Sleep. 2017; 40: A105-A105.

75. Voss U, Holzmann R, Tuin I, Hobson AJ. Lucid dreaming: A state of consciousness with features of both waking and non-lucid dreaming. Sleep. 2009; 32: 1191-1200.

76. He Y, Sun N, Wang Z, Zou W. Effect of repetitive transcranial magnetic stimulation (rTMS) for insomnia: A protocol for a systematic review. BMJ Open. 2019; 9: e029206.

77. Kennedy SH, Lam RW, Mclntyre RS, Tourjman SV, Bhat V, Blier P, et al. Canadian Network for Mood and Anxiety Treatments (CANMAT) 2016 clinical guidelines for the management of adults with major depressive disorder: Section 3. Pharmacological treatments. Can J Psychiatry. 2016; 61: 540-560.

78. Nardone R, Höller Y, Brigo F, Tezzon F, Golaszewski S, Trinka E. Transcranial magnetic stimulation and sleep disorders: Pathophysiologic insights. Sleep Med. 2013; 14: 1047-1058.

79. Feng J, Zhang Q, Zhang C, Wen Z, Zhou X. The Effect of sequential bilateral low-frequency rTMS over dorsolateral prefrontal cortex on serum level of BDNF and GABA in patients with primary insomnia. Brain Behav. 2019; 9: e01206.

80. Jiang CG, Zhang $\mathrm{T}$, Yue FG, Yi MI, Gao D. Efficacy of repetitive transcranial magnetic stimulation in the treatment of patients with chronic primary insomnia. Cell Biochem Biophys. 2013; 67: 169-173.

81. Kunze T, Langguth B, Eichhammer P, Hajak G, Fleischmann H. rTMS for the treatment of insomnia-two case reports. Psychiatr Prax. 2007; 34: S77-S78.

82. Song P, Lin H, Li S, Wang L, Liu J, Li N, et al. Repetitive transcranial magnetic stimulation (rTMS) modulates time-varying electroencephalography (EEG) network in primary insomnia patients: A TMS-EEG study. Sleep Med. 2019; 56: 157-163.

83. Yu H, Zheng W, Liu M, Ding W, Xu G, Guo L. Effects of magnetic stimulation on insomnia based on brain functional networks. IEEE Trans Magn. 2018; 54: 1-4.

84. Zhang YP, Liao WJ, Xia WG. Effect of acupuncture cooperated with low-frequency repetitive transcranial magnetic stimulation on chronic insomnia: A randomized clinical trial. Curr Med Sci. 2018; 38: 491-498. 
85. Nofzinger EA. What can neuroimaging findings tell us about sleep disorders? Sleep Med. 2004; 5: S16-S22.

86. Huang Z, Li Y, Bianchi MT, Zhan S, Jiang F, Li N, et al. Repetitive transcranial magnetic stimulation of the right parietal cortex for comorbid generalized anxiety disorder and insomnia: A randomized, double-blind, sham-controlled pilot study. Brain Stimul. 2018; 11: 1103-1109.

87. Jiang B, He D, Gao Z. Efficacy and placebo response of multimodal treatments for primary insomnia: A network meta-analysis. Clin Neuropharmacol. 2019; 42: 197-202.

88. Guo F, Lou J, Han X, Deng Y, Huang X. Repetitive transcranial magnetic stimulation ameliorates cognitive impairment by enhancing neurogenesis and suppressing apoptosis in the hippocampus in rats with ischemic stroke. Front Physiol. 2017; 8: 559.

89. Baruth JM, Casanova MF, El-Baz A, Horrell T, Mathai G, Sears L, et al. Low-frequency repetitive transcranial magnetic stimulation modulates evoked-gamma frequency oscillations in autism spectrum disorder. J Neurother. 2010; 14: 179-194.

90. Moisset $X$, de Andrade DC, Bouhassira D. From pulses to pain relief: An update on the mechanisms of rTMS-induced analgesic effects. Eur J Pain. 2016; 20: 689-700.

91. McAllister A. Neurotrophins and neuronal differentiation in the central nervous system. Cell Mol Life Sci. 2001; 58: 1054-1060.

92. Antal A, Herrmann CS. Transcranial alternating current and random noise stimulation: Possible mechanisms. Neural Plast. 2016; 2016: 3616807.

93. Mensen A, Gorban C, Niklaus M, Kuske E, Khatami R. The effects of theta-burst stimulation on sleep and vigilance in humans. Front Hum Neurosci. 2014; 8: 420.

94. O’byrne J, Rosa MB, Gouin J-P, Dang-Vu T. Neuroimaging findings in primary insomnia. Pathol Biol. 2014; 62: 262-269.

95. Yu S, Guo B, Shen Z, Wang Z, Kui Y, Hu Y, et al. The imbalanced anterior and posterior default mode network in the primary insomnia. J Psychiatr Res. 2018; 103: 97-103.

96. Poreisz C, Boros K, Antal A, Paulus W. Safety aspects of transcranial direct current stimulation concerning healthy subjects and patients. Brain Res Bull. 2007; 72: 208-214.

97. Matsumoto H, Ugawa Y. Adverse events of tDCS and tACS: A review. Clin Neurophysiol Pract. 2017; 2: 19-25.

98. Machii K, Cohen D, Ramos-Estebanez C, Pascual-Leone A. Safety of rTMS to non-motor cortical areas in healthy participants and patients. Clin Neurophysiol. 2006; 117: 455-471.

99. Association of Food \& Drug Officials of the United States. Appraisal of the safety of chemicals in foods, drugs, and cosmetics. United States; 1959.

100. Laakso I, Mikkonen M, Koyama S, Hirata A, Tanaka S. Can electric fields explain interindividual variability in transcranial direct current stimulation of the motor cortex? Sci Rep. 2019; 9: 626.

101. Antonenko D, Thielscher A, Saturnino GB, Aydin S, Ittermann B, Grittner U, et al. Towards precise brain stimulation: Is electric field simulation related to neuromodulation? Brain Stimul. 2019; 12: 1159-1168. 


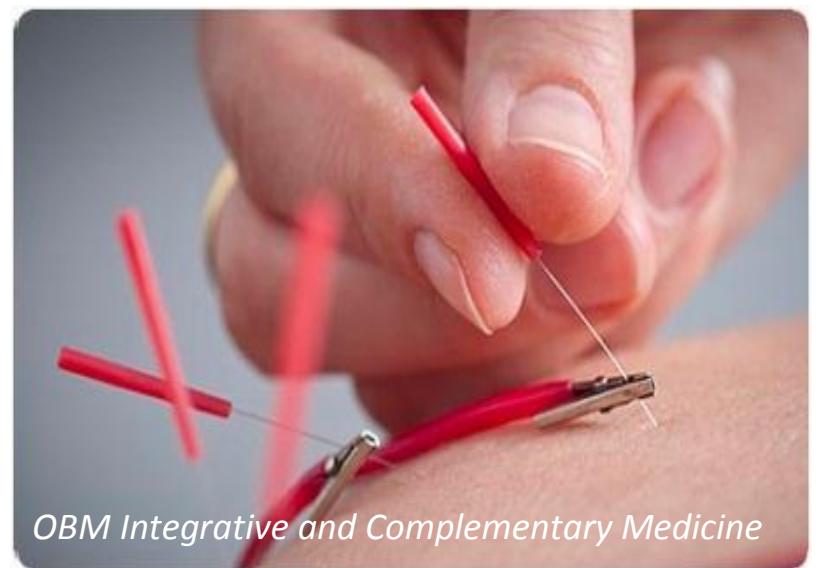

Enjoy OBM Integrative and Complementary Medicine by:

1. Submitting a manuscript

2. Joining in volunteer reviewer bank

3. Joining Editorial Board

4. Guest editing a special issue

For more details, please visit:

http://www.lidsen.com/journals/icm 